\title{
Relation between families of granules, mesogranules and photospheric network
}

\author{
Th. Roudier and R. Muller \\ Laboratoire d'Astrophysique de Toulouse, Observatoire Midi-Pyrénées, 57 avenue d'Azereix 65008 Tarbes, France \\ Received 25 November 2003 / Accepted 13 February 2004

\begin{abstract}
The analysis of a solar granulation 3-h time sequence obtained at the Pic du Midi Observatory confirms the existence of Trees of Fragmenting Granules (TFG). The $T F G$ lifetime histogram is fitted by a power law with an exponent equal to -1.68 . The positive divergences (i.e. mesogranules) come from several families that are visible at different stages of the mesogranule evolution. We observe a good coincidence between the cork (passive scalars) distribution and the $T F G$ boundaries. Thus, $T F G$ s seem to play a role in the diffusion of the magnetic elements on the Sun surface.
\end{abstract}

Key words. Sun: photosphere - Sun: granulation

\section{Introduction}

The magnetic field in the quiet sun is composed in part of discrete flux tubes which form the well-known photospheric network. These flux tubes are buffeted and deformed continuously by granulation and diffuse towards the boundaries of the supergranule. The mechanism which drives the diffusion of the magnetic field towards the supergranule borders is not well understood. In the classical view there are three convective scales, granulation, mesogranulation and supergranulation, which are produced by the de-ionization of hydrogen or helium (Unsöld 1930; November et al. 1981; Simon \& Weiss 1968). Simulations have shown that the combination of the motions generated by these three convective scales is a possible mechanism of diffusion of the magnetic elements towards the supergranule limits (Simon et al. 1991).

In recent work a different explanation has been presented on the origin of the meso and supergranulation. Rieutord et al. (2000) have proposed that, in all likelihood, mesogranulation is not a true scale of solar convection, but the combination of the effects of both highly energetic granules and data processing. According to these authors the supergranulation is the result of a nonlinear large-scale instability of the granular flow, triggered by exploding granules. Similarly, Rast (2003) claims that the scale of supergranulation has its origin in the interaction and merging of individual granular plumes. To understand the faster rotation of the supergranulation relative to the magnetic features, Gizon \& Duvall (2003) suggest a wavelike nature of the supergranulation. It is therefore interesting to revisit the interaction of the various dynamical scales on the sun surface which can advect the flux tubes.

Send offprint requests to: Th. Roudier,

e-mail: roudier@obs-mip.fr
In this paper, we will limit our study to mesoscale evolution and interactions. Recently, Roudier et al. (2003) have shown that a significant fraction of the granules in the photosphere is organized in families of granules which are named "Trees of Fragmenting Granules" (TFGs). A $T F G$ consists of a family of repeatedly splitting granules, originating from a single granule at its beginning. The TFGs can live much longer (up to $8 \mathrm{~h}$ ) than individual granules $(10 \mathrm{~min})$. When averaged in time, such long-lived TFGs produce coherent diverging flows which are identified as mesogranules. In the following, long-lived $T F G$ s are defined as $T F G$ s with lifetime greater than $1 \mathrm{~h} 30$.

The aim of the present paper is to determine the relations between the divergence of the flow vector associated with mesogranulation, the TFGs and Network Bright Points (NBPs). For this purpose, we use the data that have been analyzed by Muller et al. (1992), where mesogranule identified as the positive divergences move towards supergranule boundaries.

\section{Data}

The granulation movie used for our investigation was obtained with the $50 \mathrm{~cm}$ refractor of the Pic du Midi Observatory, on September 20, 1988. It consists of 526 white light (5750 ̊) frames taken every $20 \mathrm{~s}$. Each was selected in a burst of 50 frames. Most selected frames are of very good quality and near the diffraction limit $\left(00^{\prime} 25\right)$. The field of view is $100^{\prime \prime} \times$ $70^{\prime \prime}$ and the pixel size is $00^{\prime} 14$. The photographs were digitized with a $1024 \times 1024$ CCD camera at Lockheed Palo Alto Laboratory and then the frames were processed: aligned, destreched and finally filtered to remove 5-min p-mode oscillations. Our analysis is restricted to an area of $58^{\prime \prime} \times 48^{\prime \prime}$ common to all the frames, which includes a large part of a supergranule. The boundary of the supergranule is clearly 


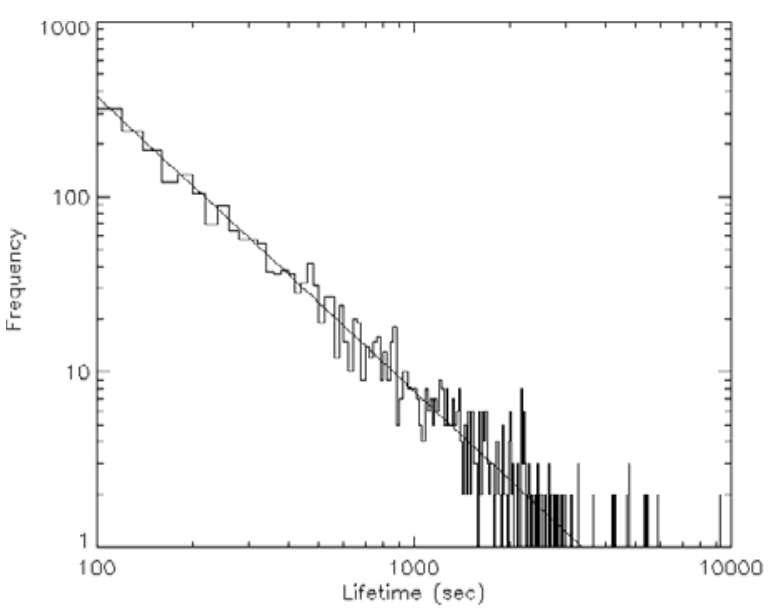

Fig. 1. Lifetime histogram of individual granules and $T F G$ s. It is well fitted by a power law with an exponent equal to -1.68 .

outlined by the converging flows of granules and by the location of Network Bright Points (Muller et al. 1992).

\section{TFGs detection and lifetime}

The method of detecting families of splitting granules (TFGs) has been described in Roudier et al. (2003). To summarize, the intensity map are transformed into a binary maps using the local curvature of the intensity field (Strous 1994). When the second spatial derivatives have the same sign in the four directions, for example negative, the pixel belongs to a bright object (granule), in the opposite case it belongs to a dark intergranule. Once the segmentation is performed the objects are labelled. The evolution of the granules is tracked by identifying the temporal links in the 3D data box (2D in space and 1D in time). All the granules originating from a single progenitor, by splitting in sucessive generations, are labelled with the same label as the progenitor. The end of a family is determined when the last granules of the family dilute in most cases or merge in a granule with a larger area. This is the criterium to label in time the $T F G$ in the case of merging.

We constructed in this way 10744 objects (the term object stands for a set of granules with the identical label) contained within the 3D $(x, y, t)$ box. Among those, only 7471 had a lifetime greater than one frame. Of those 7471 objects, 512 had at least one splitting and formed the set of what we call "Trees of Fragmenting Granules" (TFGs). The remaining 6959 objects are called individual granules.

The lifetime histogram of individual granules and TFGs (Fig. 1) is well fitted by a power law $N \propto t^{\gamma}$, with $\gamma=-1.68$ which is very close to the exponent found in Roudier et al. (2003) $\gamma=-1.72$. Such fit indicates that we cannot give a characteristic lifetime to the TFGs.

\section{Relation between mesogranules and TFGs}

It has been shown in Roudier et al. (2003) that horizontal flow fields of long-lived $T F G$ computed by using only the granules belonging to that family, produce coherent diverging

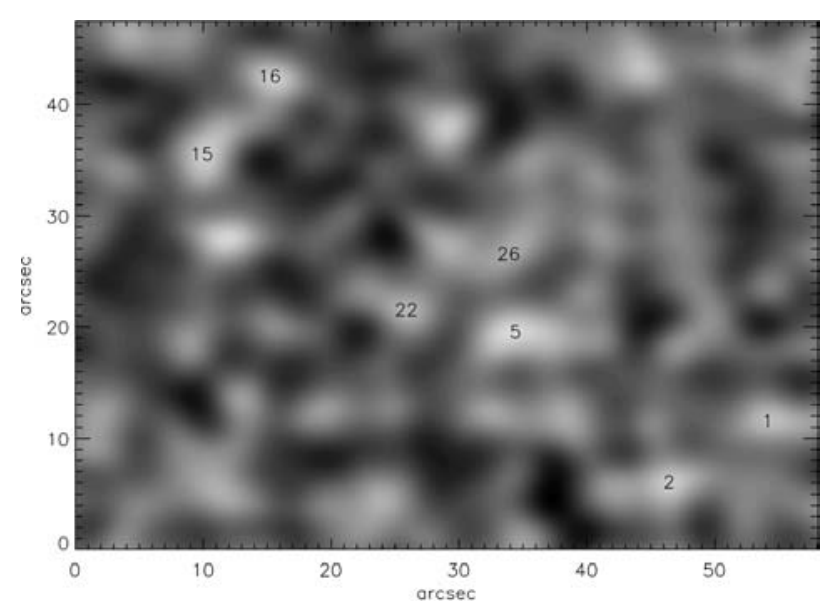

Fig. 2. Flow divergence $(t=9 \mathrm{~min})$ identical to Fig. 2 first image left column of Muller et al. (1992). Mesogranule numbers are indicated over the positive divergence selected by Muller et al. (1992).

flows. These coherent diverging flows have been identified as mesogranules.

In this paper we extend the study of the relation between mesogranules and TFGs which was restricted up today to long-lived TFGs. The mesogranules detected as divergences by Muller et al. (1992) are used for that purpose. To detect the same features, we have computed here the divergence of the flow field with the same temporal and spatial windows, $17 \mathrm{~min}$ and 3". $3 F W H M$, respectively. They are shown in Fig. 2 at the same instant as in Fig. 2 (first top left) of Muller et al. (1992), and with the same labels. The windows parameters have been chosen to remove the flow pattern produced by individual granules. Thus, for each mesogranule identified in Fig. 2, we determined the granules and family numbers.

Figure 3 shows different families with the flow field at the same location. Some divergences visible in Fig. 2 are indicated by circles in Fig. 3. At these locations, we observe positive diverging flow associated with $T F G$ s and confirm the result of Roudier et al. (2003).

In the following, we describe the most representative examples of the links between divergences and TFGs.

In the first divergence map of Fig. 4 ( $t=9 \mathrm{~min}$; top left), the divergence labelled 5 corresponds to four families This is not surprising because the granule labeling of the first frame of our observation does not allow us to take care of the full granule history. So the detected divergence 5 at the beginning of the observation cannot rule out the link between the TFGs and the divergence. But during the time evolution of the divergence, new families are growing inside the mesogranule identified by the number 5. As shown in Fig. 4 at $t=92 \mathrm{~min}$, a new family is detected. This new family then pushes the old ones. This event repeats at $t=171 \mathrm{~min}$. Particular care has been taken to verify that the families are new ones. So Muller's mesogranule 5 which is identified as the same structure during the time sequence, corresponds in fact to the evolution of different families, unresolved by the spatial (3"'3) and temporal (17 min) windows. 


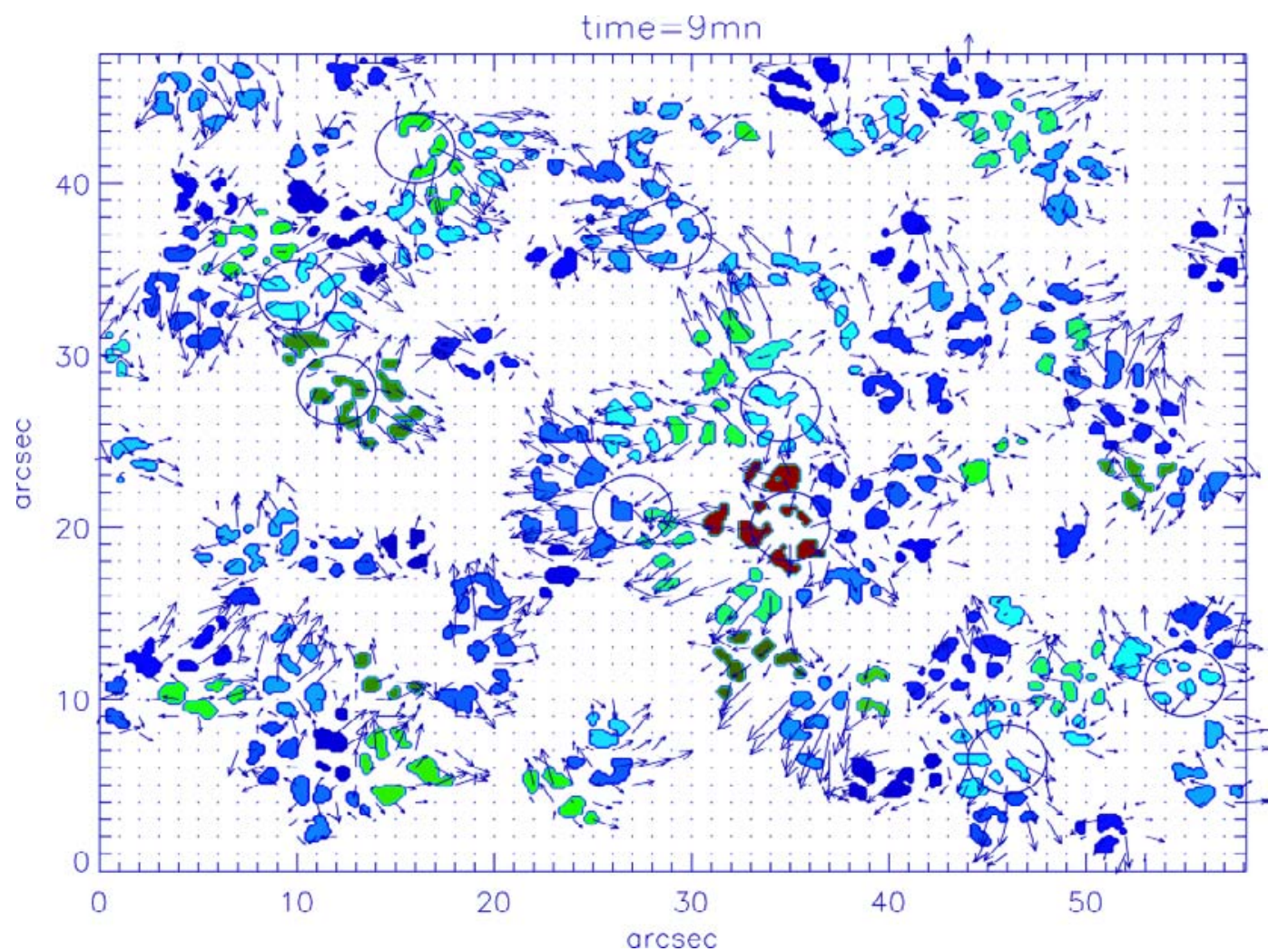

Fig. 3. Families $(T F G s>7200$ s) together with the flow field at the same location. Circles indicate the location of some divergence visible in Fig. 2.

Table 1. Number of families (lifetime $>7200 \mathrm{~s}$ ) found in each mesogranule during its life (i.e. Fig. 2).

\begin{tabular}{llllcccc}
\hline \hline Divergence & 1 & 2 & 5 & 15 & 16 & 22 & 26 \\
\hline No. of fam. & 2 & 5 & 6 & 2 & 4 & 3 & 7 \\
\hline
\end{tabular}

Thus, the motion described in Muller et al. (1992) may appear as the combination of the evolution of different families and not as the motion of the same entity.

Another example is divergence number 15. It is quite stable during the time sequence; it is produced most of the time by only one family. An extension of Muller's mesogranule 15 is due to a close second family ( $t=117 \mathrm{~min}$ ). This is also the case of the divergence number 2 which is composed of a family visible during all the time sequence and four adjacent families which appear at different times.

More generally, several long-lived families evolve in the divergences taken into consideration in Muller et al. (1992), which are among the largest and strongest detected in the field of view. Table 1 summarize the number of long-lived families for each divergence. In addition to the long-lived families, many shorter families appear and disappear, contributing to the divergence. A family of granules has its own identity. The spatial extension and the lifetime of the pattern depends of the characteritics (time averaging and spatial smoothing) of the windows to detect them. When $17 \mathrm{~min}$ and 3 .' 3 windows are used, several families are associated with the largest divergences. This confirms previous results of Rieutord et al. (2000) who show that the increase of the size of windows tends to mix structures which are here the TFGs. The use of, divergence quantity is not suited to keep the temporal link of the positive divergence. On the contrary the temporal evolution of structure is followed by using the TFGs.

\section{TFG proper motion}

To describe the evolution and the interactions of the long-lived $T F G$ s (lifetime $>7200 \mathrm{~s}$ ), we plotted the cumulative view of the $T F G$ s at different times. The cumulative view has the advantage of showing directly the dominant structures, here $T F G$ s, at each step of the time evolution in the field of view. The evolution is shown in Fig. 5 where the cumulative displacements of the centre of gravity of the families have been overplotted. Then depending of their locations, we observe a larger TFG displacement at mean distance between the centre and the limit of the supergranule outlined by the NBPs in Fig. 6. In the center of each panel of Fig. 5 we note that the motions of the TFGs are limited by their environment. Regarding the direction of their displacement, we observe that some of them change direction during their life (see encircled TFG in Fig. 5). However, displacements at the beginning of the sequence are more noisy due to the shorter time interval and they are probably influenced by older TFGs born before the beginning of the sequence. The movie of the long-lived TFGs shows that all families adjust their development relative to their neighbouring family, the most energetic ones dictating the evolution of 

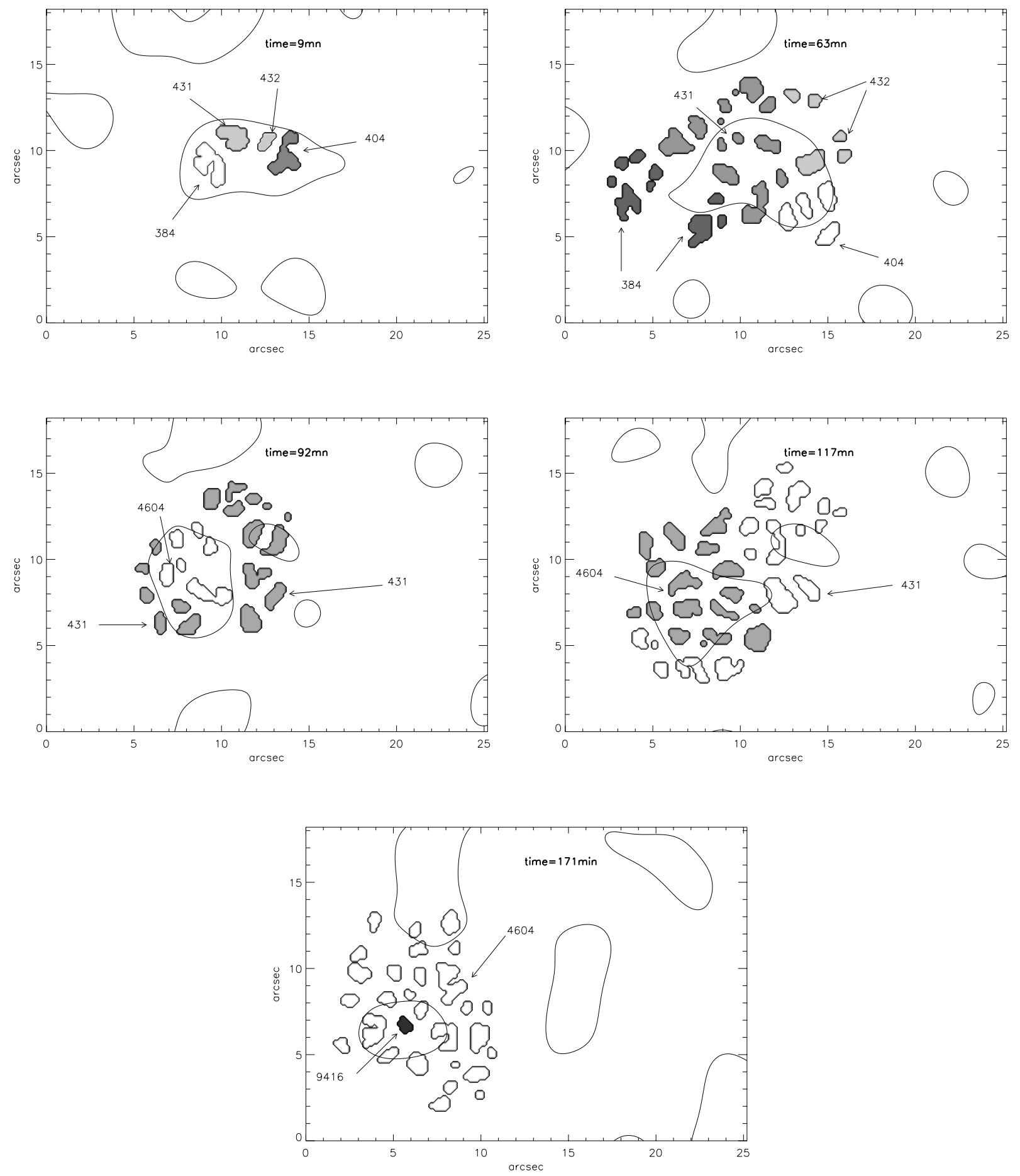

Fig. 4. Time evolution of the divergence 5 of Muller et al. (1992), and the corresponding $T F G$ s located in the area of this divergence. TFG numbers are indicated on each map.

others. This is similar to the description of the granule evolution given by Schriver et al. (1997).

At the end of our time sequence, we observe that long-lived families (lifetime $>7200 \mathrm{~s}, 2 \mathrm{~h}$ ) cover pratically all the area and $T F G$ displacements exhibit the large scale pattern which can be associated with the supergranular flows. The associated velocities of the centre of gravity of the TFGs lies between 0 and $0.8 \mathrm{~km} \mathrm{~s}^{-1}$ with a peak around $0.3 \mathrm{~km} \mathrm{~s}^{-1}$. The divergence motions measured by Muller et al. (1992) are similar to those shown in Fig. 5. The similarity of their motions confirms the close relation between families and divergences described in the previous section and in Roudier et al. (2003). Then both long-lived TFGs and centres of divergence appear to be advected by supergranular flow.

Families of shorter lifetimes behave like the longer ones; they adjust their development relative to their environment and they move coherently with the long-lived family, toward the boundary of the supergranule. 

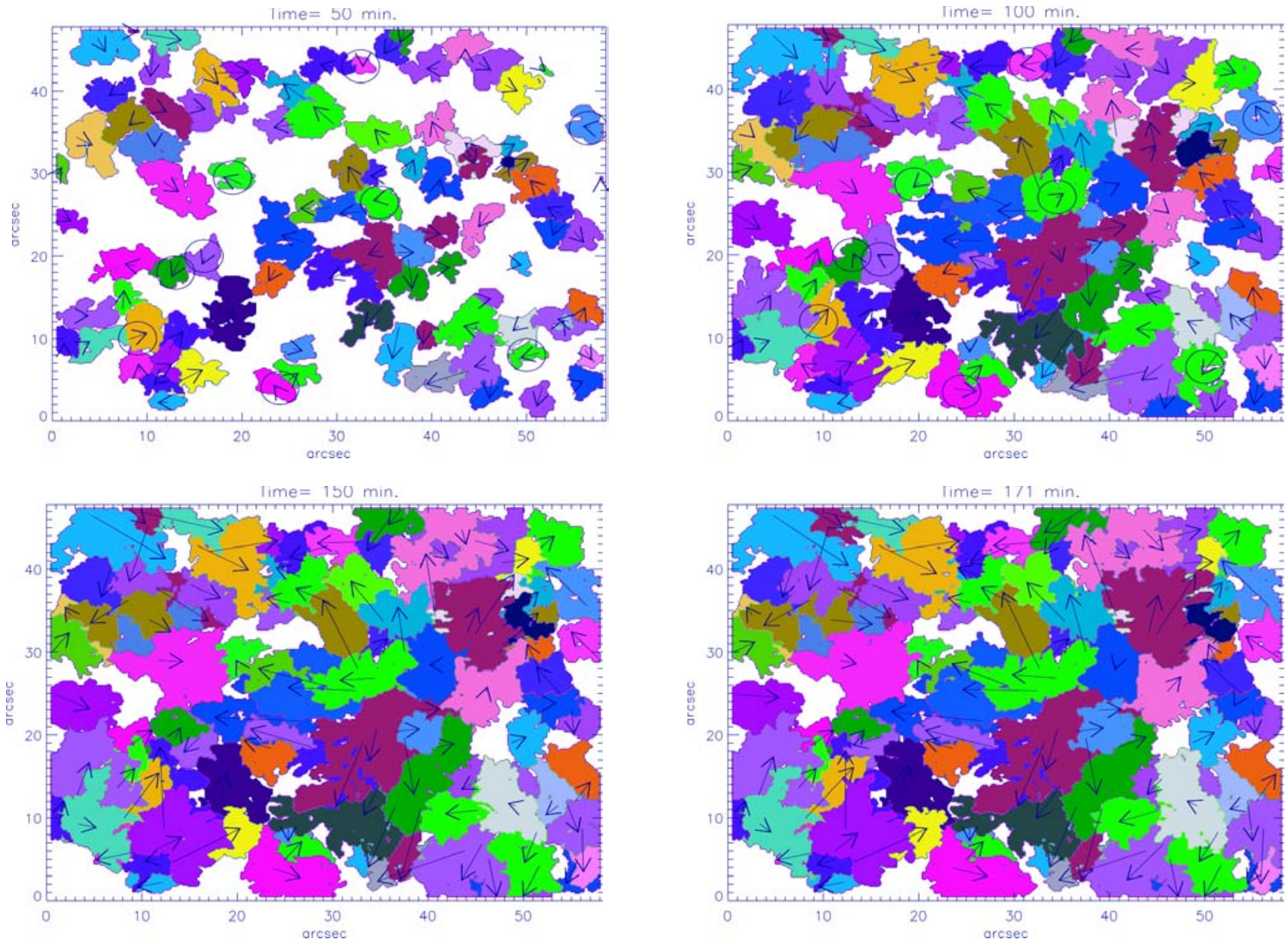

Fig. 5. Time evolution of the $T F G$ s overlapped with the displacement of their centre of gravity. Each $T F G$ displacement represents the cumulative displacement between the beginning of the time sequence and the time of each plot. Encircled $T F G$ s are those which change direction during their lifetime.

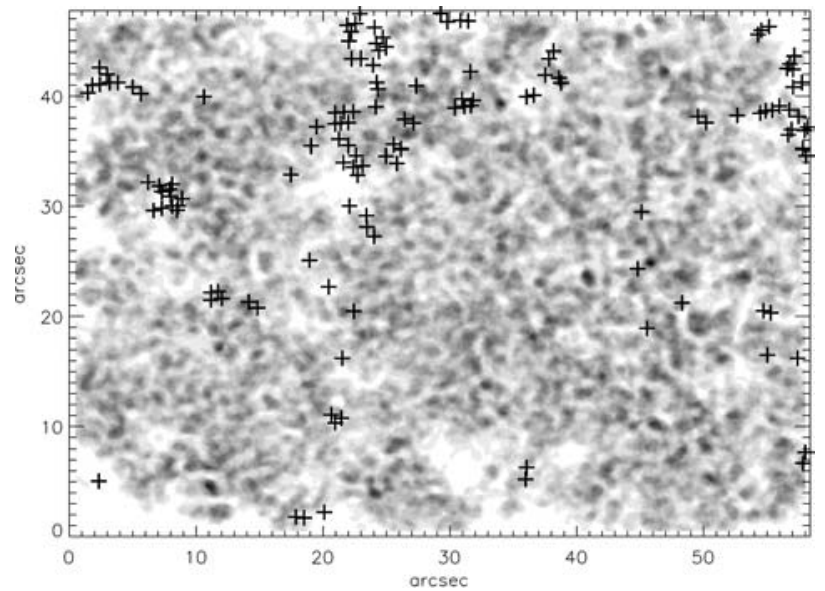

Fig. 6. NBP location (cross) and the density of $T F G s>5400$ s. The density of $T F G$ s is proportional to the brightness in the image.

\section{Relation between Network Bright Points and TFGs}

In Fig. 6, we plotted all the Network Bright Points (NBPs) identified in our 3 -h time series and the sum of all the granules belonging to the families of granules living more than $5400 \mathrm{~s}$.

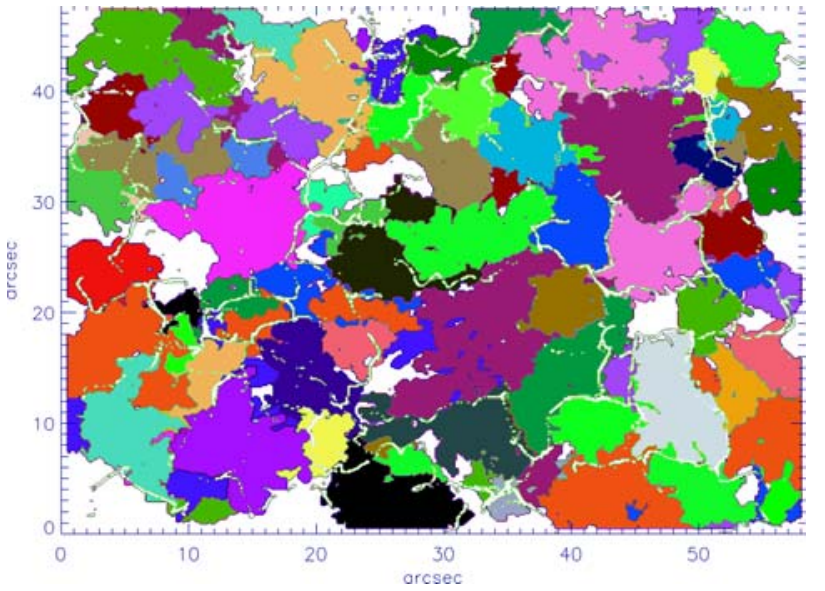

Fig. 7. Cumulative view of the $T F G$ s at the end of the time sequence and the final corks distribution after three hours of advection.

The NBPs outline roughly the boundary of a supergranule (Muller et al. 1992). In Fig. 6 the location of the NPBs coincides with the reduced density of long lived-families. Moreover, at the supergranule boundary are either short-lived or simply missing. 


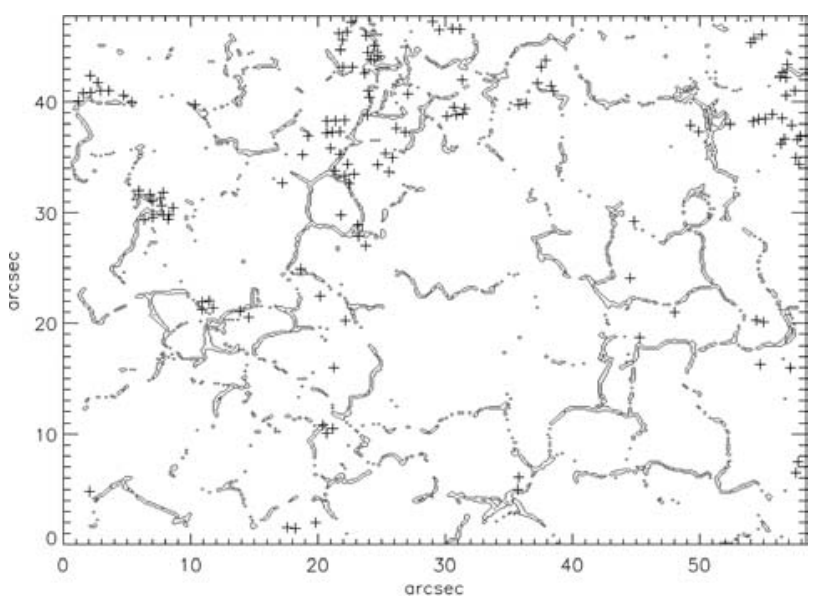

Fig. 8. Location of the NBPs (cross) and cork at the end of the time sequence.

One way to know more about the role of $T F G$ s in the diffusion of the magnetic elements is to compute the advection of floating corks by the granular flow. This technique was already used by Simon et al. (1991). It consists of following the trajectories of floating corks, initially uniformly distributed, and characterizing their spatial distribution after some time. Here we use the techniques described in Roudier et al. (1999). We determine the velocity field evolution of the Pic du Midi data set with a high spatial and temporal resolution ( $0 . ' 7$ and $5 \mathrm{~min})$. We then use this field to compute the cork trajectories during the three hours of recorded data. Figure 7 shows the cumulative view of the TFGs at the end of the time sequence and the final cork distribution after three hours of advection. As shown in this figure, corks are expelled from TFGs and concentrate in regions which coincide with limits of the TFGs. This result is quite remarkable because the computation of the velocity field which drives the corks motions and the TFG detection is independent. The coincidence of the corks distribution and the $T F G$ s indicates that $T F G$ s appear to play a role in the diffusion of the magnetic elements on the Sun surface. A previous result obtained with the same data, described in Rieutord et al. (2000), shows that corks concentrate in regions that coincide with those occupied by network bright points which delineated the supergranulation scale (Fig. 8). This suggests a key role of the TFGs in the building of the photospheric magnetic network in the quiet Sun.

\section{Conclusion}

From the analysis of the solar granulation 3-h time sequence obtained at the Pic du Midi Observatory, we confirm the existence of TFGs. We find a remarkable fitting of the TFG lifetime histogram of individual granules by a power law whose exponent is identical to the one found by Roudier et al. (2003). We confirm also the link between TFGs and positive divergences. The detailed analysis of this relation shows that divergences come from several families that are visible at different stages of the evolution. The spatial (3".3) and temporal (17 min) windows used in Muller et al. (1992) to identify divergences (i.e. mesogranules) were selected to remove the granulation scale.
In this way they observed scales larger than granulation but with the tendency to mix structures, namely the TFGs.

The TFGs adjust their development relative to their family neighbours, the more energetic ones dictating the evolution of the others. The long-lived TFGs investigated here move like the divergences analysed by Muller et al. (1992): they move outward from the centre to the boundary of the supergranule, at supergranular speed, when they are located at mid-distances; they are more stationary when they evolve close to the centre or to the boundary. Thus both long-lived TFGs and divergence appear to be advected by supergranular flow.

The NBPs are located where the density of long-lived family of granules is in general low at the limit of the supergranule. The corks which are used as passive scalars are expelled from $T F G$ s and concentrate in regions which coincide with the limits of the TFGs. This result is quite remarkable because the computation of the velocity field that drives the cork motion and the TFG detection are independent. This result must be compared with the persistent magnetic pattern found by Dominguez Cerdeña et al. (2003), which is visible at the spatial scale of the mesogranulation. The coincidence of the cork distribution and the TFG limits indicates that $T F G$ s seem to play a role in the diffusion of the magnetic elements on the Sun surface.

While $T F G$ 's displacements exhibit large scale pattern that can be associated with the supergranule flows, some questions arise from our analysis, for instance:

- what is the degree of contribution of the TFGs to the diffusion of magnetic field?

- how do TFGs contribute to the building of the photospheric network?

The answer to such questions only will be possible by developing simulations in which the different sources, at different scales, will be easily identified.

Acknowledgements. We would like to thank very much Jose Antonio Bonet for very fruitful and helpful comments on the manuscript.We thank Michel Rieutord for useful advice and comments. This work was supported by the Centre National de la Recherche Scientifique (CNRS, UMR 5572) and the Programme National Soleil Terre (PNST). Special thanks are due to the Pic du Midi Observatory staff for their technical assistance.

\section{References}

Dominguez Cerdeña, I., Sanchez Almedia, J., \& Kneer, F. 2003, A\&A, 407, 741

Gizon, L., \& Duvall, T. L. 2003, SOHO 12/GONG, ESA SP-517

Muller, R., Auffret, H., Roudier, Th., et al. 1992, Nature, 356, 322

November, L. J., Toomre, J., Gebbie, K. B., \& Simon, G. W. 1981, ApJ, 245, 12

Rast, M. P. 2003, SOHO 12/GONG, ESA SP-517

Roudier, T., Lignieres, F., Rieutord, M., Brandt, P. N., \& Malherbe, J. M. 2003, A\&A, 409, 299

Rieutord, M., Roudier, T., Malherbe, J. M., \& Rincon, F. 2000, A\&A, 357,1063

Schrijver, C. J., Hagenaar, H. J., \& Title, A. M. 1997, ApJ, 475, 328

Simon, G. W., \& Weiss, N. O. 1968, Zeit. Astrophys., 69, 435

Simon, G. W., Title, A. M., \& Weiss, N. O. 1991, ApJ, 375, 775

Strous, L. H. 1994, Ph.D. Thesis (Utrecht)

Unsöld, A. 1930, Z. Astrophys., 1, 138 